\title{
Cytoprotective Mitochondrial Chaperone TRAP-1 As a Novel Molecular Target in Localized and Metastatic Prostate Cancer
}

\author{
Irwin Leav, ${ }^{*}$ Janet Plescia, ${ }^{*}$ Hira Lal Goel, ${ }^{*}$ \\ Jing $\mathrm{Li},{ }^{*}$ Zhong Jiang, ${ }^{\dagger}$ Ronald J. Cohen, ${ }^{*}$ \\ Lucia R. Languino, ${ }^{*}$ and Dario C. Altieri ${ }^{\star}$ \\ From the Prostate Cancer Discovery and Development Program,* \\ Department of Cancer Biology, and the Department of \\ Pathology, ${ }^{\dagger}$ University of Massachusetts Medical School, \\ Worcester, Massachusetts; and Uropath Pty Ltd., \\ Nedlands, Australia
}

Molecular chaperones of the heat shock protein-90 (Hsp90) family promote cell survival, but the molecular requirements of this pathway in tumor progression are not understood. Here, we show that a mitochondrialocalized Hsp90 chaperone, tumor necrosis factor receptor-associated protein-1 (TRAP-1), is abundantly and ubiquitously expressed in human high-grade prostatic intraepithelial neoplasia, Gleason grades 3 through 5 prostatic adenocarcinomas, and metastatic prostate cancer, but largely undetectable in normal prostate or benign prostatic hyperplasia in vivo. Prostate lesions formed in genetic models of the disease, including the transgenic adenocarcinoma of the mouse prostate and mice carrying prostate-specific deletion of the phosphatase tensin homolog tumor suppressor $\left(\right.$ Pten $\left.^{\mathrm{pc}^{\mathrm{C}}-/}\right)$, also exhibit high levels of TRAP-1. Expression of TRAP-1 in nontransformed prostatic epithelial BPH-1 cells inhibited cell death, whereas silencing of TRAP-1 in androgen-independent PC3 or DU145 prostate cancer cells by small interfering RNA enhanced apoptosis. Targeting TRAP-1 with a novel class of mitochondria-directed Hsp90 inhibitors, ie, Gamitrinibs, caused rapid and complete killing of androgen-dependent or -independent prostate cancer, but not BPH-1 cells, whereas reintroduction of TRAP- 1 in BPH-1 cells conferred sensitivity to Gamitrinib-induced cell death. These data identify TRAP-1 as a novel mitochondrial survival factor differentially expressed in localized and metastatic prostate cancer compared with normal prostate. Targeting this pathway with Gamitrinibs could be explored as novel molecular therapy in patients with advanced prostate cancer. (Am J Pathol 2010, 176:393-401; DOI: 10.2353/ajpath.2010.090521)

Apart from skin tumors, prostate cancer is the most commonly diagnosed malignancy in men in the United States. ${ }^{1}$ Despite progress in early diagnosis, ${ }^{2}$ and prolongation of patient survival, ${ }^{3}$ the disease still carries significant morbidity and mortality, with its advanced and metastatic phase claiming over 30,000 deaths per year in the United States alone. Similar to the genetic heterogeneity of most epithelial malignancies, prostate cancer progresses through a stepwise acquisition of multiple molecular changes, ${ }^{4}$ of which insensitivity to androgen deprivation, ${ }^{5}$ emergence of an 'osteomimetic' phenotype responsible for metastatic tropism to the bone, ${ }^{6}$ and deregulated cell proliferation and cell survival, ${ }^{7}$ are pivotal traits.

In this context, advanced prostate cancer is almost invariably associated with a heightened anti-apoptotic threshold, ${ }^{4}$ which may contribute to disease progression and resistance to therapy. This process often involves aberrant resistance to mitochondrial cell death, ${ }^{8}$ with reduced organelle permeability to solutes, and attenuated release of mitochondrial apoptogenic proteins in the cytosol. ${ }^{9}$ The regulators of such 'mitochondrial permeability transition' normally triggered by cell death stimuli are still largely elusive, but knockout data in mice have identified pro-apoptotic Bcl-2 family proteins and the mitochondrial matrix immunophilin, cyclophilin D, as pivotal effectors of this process, controlling the integrity of the mitochondrial outer membrane, $^{8}$ and the opening a permeability transition pore, ${ }^{10,11}$ respectively.

Recent data have shown that molecular chaperones of the heat shock protein-90 (Hsp90) family, ${ }^{12}$ may function

Supported by NIH grants CA89720 and CA109874 (to L.R.L.), and CA78810, CA90917, and CA118005 (to D.C.A.).

Accepted for publication September 8, 2009

Address reprint requests to Dario C. Altieri, M.D., Department of Cancer Biology, LRB428, University of Massachusetts Medical School, 364 Plantation Street, Worcester, MA 01605. E-mail: dario.altieri@umassmed.edu. 
as novel regulators of mitochondrial permeability transition, ${ }^{13}$ especially in tumor cells. ${ }^{14}$ Accordingly, Hsp90, and its ortholog, tumor necrosis factor receptor-associated protein-1 (TRAP-1) are abundantly localized to mitochondria of tumor, but not most normal cells, and antagonize cyclophilin D-dependent pore-forming function, potentially via a protein (re)folding mechanism. ${ }^{14}$ Consistent with a general role of Hsp90 as a drug target in prostate cancer ${ }^{15}$ this mitochondria-compartmentalized cytoprotective pathway could provide a novel therapeutic target to enhance tumor cell apoptosis. ${ }^{14}$

In the current study, we demonstrate that TRAP-1 is dramatically expressed in all lesions that comprise the entire natural history of human prostate cancer, as well as genetic disease models in rodents, but undetectable in the normal prostate. Importantly, we show that Gamitrinibs, a novel class of small molecule Hsp90 antagonists selectively engineered to target the pool of these chaperones in mitochondria, ${ }^{16}$ cause sudden prostate cancer cell death without affecting nontransformed prostatic epithelium.

\section{Materials and Methods}

\section{Patient Population and Characteristics of Tissue Samples}

Forty cases of archived paraffin tissue blocks of radical prostatectomies were selected from patients with localized cancer. The patients ranged in age from 52 to 75 years (mean 64). None of the 40 men had received any hormonal or radiation therapy before surgery. Of the 40 cases of localized prostate cancer, $5 \%$ had a total Gleason grade score of 6 , whereas $76 \%$ had a total Gleason grade score of 7 . Of these, Gleason grade 3 predominated in $50 \%$ of the cases, while Gleason grade 4 cancers constituted the major pattern in the remaining cases. In four of these latter grades, significant areas of intraductal carcinoma, considered to be a variant of Gleason grade 4 or $5,{ }^{17}$ were also evident. Nine of the 40 cases had total Gleason scores of 8 to 10, in which foci of Gleason grade 5 cancers were detected, admixed with those of lower grades.

To evaluate metastatic disease, three cases of prostatic adenocarcinoma metastasized to pelvic lymph nodes and two cases of prostate cancer metastases to bones were selected. The lymph node metastases were obtained from untreated patients, whereas the two bone biopsy samples came from men that received anti-androgen therapy for 5 and 8 months, respectively.

For analysis of normal prostate, benign glands mixed with cancerous lesions, or foci of benign prostatic hyperplasia (BPH) admixed in eight cancer cases were studied. In addition, four tissue samples of normal prostate collected at autopsy from the central zone (two cases) or peripheral zone (two cases) of men 58 to 70 years of age were collected, and compared with samples of BPH foci and/or benign glands in the context of cancerous lesions.

The patient series used in this study was collected from the Department of Pathology at the University of
Massachusetts Medical School, the collection of Dr. John $\mathrm{McNeal}$ in the department of Urology at Stanford University Medical School, and Uropath PTT LtD., West Leederville, Australia.

\section{Mouse Models of Prostate Cancer}

All experiments involving animals were approved by an Institutional Animal Care and Use Committee. The transgenic adenocarcinoma of the mouse prostate (TRAMP) model has been described, ${ }^{18}$ and involves expression of the SV40 large T and small $t$ oncogene in the prostatic epithelium under the control of the minimal $-426 /+28$ rat probasin promoter. Transgene expression is regulated by androgen, thus correlates with sexual maturity, and results in the appearance of a spectrum of lesions, including prostatic intraepithelial neoplasia (PIN), invasive adenocarcinoma and metastasis. ${ }^{18}$ Female TRAMP mice on a C57BL/6 background were bred with nontransgenic males, and the offspring was weaned at 3 to 4 weeks of age. Male pups were genotyped by PCR amplification of tail genomic DNA using transgene-specific primers. For analysis of TRAP-1 expression during TRAMP carcinogenesis, mice were euthanized at 22 to 26 weeks of age, and the urogenital tract including bladder, seminal vesicles, and prostate was collected and fixed in formalin for further pathological evaluation.

For a second genetic model of prostate cancer, mice expressing a conditional deletion of the PTEN tumor suppressor in the prostatic epithelium $\left(P_{t e n}{ }^{p c-l-}\right)$ were used. ${ }^{19}$ For these experiments, male Pten ${ }^{\text {LoxP/+ }} / \mathrm{PB}-\mathrm{Cre} 4$ transgenic mice were crossed to Pten ${ }^{\text {LoxP/LoxP }}$ females to generate both $P$ ten ${ }^{L O X P / L O X P} / P B-C r e 4$ male mice, $\left(P t e n^{P C-I-}\right)$ and Pten ${ }^{\text {LoxP/LoxP }}$ non-transgenic littermates (used as control mice). The original Pten ${ }^{\text {LoxP/LoxP }}$ mouse lines were kindly provided by Dr. Hong Wu (University of California, Los Angeles), and PB-Cre4 lines were obtained from the National Cancer Institute, Bethesda, MD. Pten ${ }^{p C-1-}$ mice develop PIN lesions at 6 to 7 weeks of age (100\% penetrance), prostatic adenocarcinoma between 9 and 29 weeks (100\% penetrance), and metastases between 12 and 29 weeks (40\% penetrance). ${ }^{19}$ Although our crosses of the PB-Cre4 transgenic mice and Pten ${ }^{L O X P / L O X P}$ mice produced a Pten ${ }^{p C-1-}$ model on a mixed genetic background (C57BL/6:129SV:BALB/C), we observed negligible differences in time-dependent occurrence of PIN and prostatic adenocarcinoma in these mice, as compared with the Pten ${ }^{p C-1-}$ strain reported in the literature (C57BL/6:DBA2 crossed to 129SV:BALB/ C) ${ }^{19}$ One hundred percent of Pten ${ }^{p C-I-}$ mice used in this study exhibited high-grade PIN by 6 to 7 weeks of age. Only F2 generations of Pten ${ }^{p-1-}$ mice were used in these experiments to minimize strain-specific differences in tumor growth.

\section{Immunohistochemistry}

Five-micron sections cut from formalin-fixed, paraffinembedded human or mouse prostatic tissues were put on high adhesive slides. The various samples were 
stained with H\&E, and analyzed for differential expression of TRAP-1 by immunohistochemistry, as described. ${ }^{14}$ Briefly, tissue sections were deparaffinized, rehydrated in water, and quenched for endogenous peroxidase. Epitope heat retrieval was performed by steaming the slides in 10\% sodium citrate for 60 minutes. Processed slides were rinsed in PBS, and stained with an antibody to TRAP-1 (BD Biosciences, San Jose, CA) characterized in previous studies, ${ }^{14}$ or nonbinding $\operatorname{lgG}$, using standard avidin-biotin-peroxidase techniques (Histostain-plus, Zymed Laboratories Invitrogen, Carlsbad, CA). Slides were incubated with diaminobenzidine as a chromogen, counterstained with hematoxylin, and scored by light microscopy. The intensity of immunostaining within a specimen was given a score that ranged from $1+$ to $4+$ when compared with the expression observed in normal glands in the same tissue, or normal prostate from autopsy specimens. The scoring of immunostaining intensity is summarized and grouped as follows: Negative, - ; negligible, + ; weak, ++ ; and strong, +++ to ++++ .

\section{Molecular Targeting of Mitochondrial Hsp90 Chaperones}

Mitochondria-directed small molecule Hsp90 antagonists Gamitrinibs (GA mitochondrial matrix inhibitors) were characterized in recent studies, ${ }^{14}$ suspended in cremophor and used at concentrations of 0 to $20 \mu \mathrm{mol} / \mathrm{L}$. As reported previously, the combinatorial structure of Gamitrinibs comprises an Hsp90 inhibitory backbone derived from the chaperone ATPase antagonist, 17-allylamino geldanamycin (17-AAG) linked to 1 to 4 tandem repeats of guanidinium (Gamitrinibs G1 to G4) or triphenylphosphonium (Gamitrinib-TPP) used as mitochondria-targeting moieties. ${ }^{16}$ Non-mitochondria-targeted, ie, unconjugated 17-AAG was suspended in dimethyl sulfoxide, and used at comparable concentrations. Androgen-independent prostate adenocarcinoma PC3 and DU145, androgen-dependent LNCaP, and nontransformed prostatic epithelial BPH-1 cells were obtained from the American Type Culture Collection (Manassas, VA), and maintained in culture as recommended by the supplier. Androgenindependent and metastatic C4-2B cells were characterized previously. ${ }^{20}$

\section{Transfection Experiments}

Normal BPH-1 cells were transfected with control pcDNA or a TRAP-1 cDNA described previously, ${ }^{14}$ by Lipofectamine (Invitrogen). After 24 hours, cells were treated with the broad cell death stimulus, staurosporine (STS, 0 to $1 \mu \mathrm{mol} / \mathrm{L}$ ), which activates mitochondrial cell death, or the chemotherapeutic agent, taxol $(1 \mu \mathrm{mol} / \mathrm{L})$ for 16 hours at $37^{\circ} \mathrm{C}$, and analyzed for modulation of cell survival by an 3-(4,5-dimethylthiazol-2-yl)-2,5-diphenyltetrazolium (MTT) assay, as described, ${ }^{14}$ or generation of cleaved, ie, active caspase 3 , by Western blotting. Alternatively, $\mathrm{BPH}-1$ cells transfected with PcDNA or TRAP-1 were treated with increasing concentrations ( 0 to $20 \mu \mathrm{mol} / \mathrm{L}$ ) of Gamitrinibs, ${ }^{16}$ or unconjugated $17-A A G$, and analyzed by MTT after 6 hours. In some experiments, aliquots of DU145 cells were transfected with control non-targeting or TRAP-1-directed SMARTpool small interfering RNA (siRNA) (Dharmacon, Lafayette, Co) characterized previously ${ }^{14}$ by OligofectAmine, confirmed for protein knockdown by Western blotting after 24 to 48 hours, and analyzed for modulation of cell viability by MTT.

\section{Statistical Analysis}

Data were analyzed using the two-sided unpaired $t$-test on a GraphPad software package for Windows (Prism 4.0). A P value of 0.05 was considered as statistically significant.

\section{Results}

\section{Differential Expression of Mitochondrial TRAP-1 in Primary and Metastatic Prostate Cancer}

We began this study by examining the expression of a mitochondrial Hsp90 ortholog, TRAP- $1,{ }^{14}$ in the human prostate. The epithelium of normal prostate (peripheral or central zone) collected at autopsy, or BPH nodules, was negative for TRAP-1 expression, by immunohistochemistry (Figure 1A, Table 1). Conversely, normal prostatic glands admixed with cancer foci showed negative, negligible or weak staining for TRAP-1 (Figure 1C, Table 1). Atrophic benign glands commonly found intermixed with cancer foci were also weakly stained for TRAP-1 expression, which seem to frequently localize to basal epithelial cells, as well as regressed luminal cells (not shown).

All examples of high-grade PIN expressed TRAP-1 (Figure 1B, Table 1). In addition, all cases of Gleason grade 3 carcinoma stained positive for TRAP-1, with strong expression in over one third of the samples examined (Figure 1, B and C, Table 1). Similar results were obtained with Gleason grade 4 cancers (Figure 1D, Table 1), but in these specimens TRAP-1 reactivity was more heterogeneous, with weakly stained tumor cells intermixed with populations of more intensely labeled cells (Figure 1D). Four cases of prostatic intraductal carcinoma, considered to be variants of Gleason grade 4 or 5 cancer, ${ }^{17}$ were grouped together with grade 4 cases, and all expressed TRAP-1 to the same intensity as grade 4 cancers (Figure 1E). Gleason grade 5 cancers were also uniformly positive for TRAP-1 expression with heterogeneous staining intensity that varied from weak (Figure $1 \mathrm{~F}$ ) to strong (Figure $1 \mathrm{G}$ ) in different tumor cell populations within the various samples (Table 1).

In all strongly positive cases of PIN or prostate cancer, TRAP-1 staining appeared as discrete punctate granules dispersed throughout the cytoplasm of tumor cells (see inset panel E), potentially consistent with the subcellular localization of TRAP-1 to mitochondria. ${ }^{14}$ A control, nonbinding IgG gave no staining of normal or tumor glands, by immunohistochemistry (not shown).

Unlike the heterogeneous pattern of TRAP-1 staining in localized high-grade cancers (Table 1), immunohistochemical analysis of metastatic prostate cancer revealed uniformly intense expression of TRAP-1 in the tumor cell 



Figure 1. TRAP-1 expression in localized human prostate cancer. A: Negative TRAP-1 staining of normal prostate (peripheral zone) epithelium collected at autopsy. B: Strong staining for TRAP-1 in high-grade prostatic intraepithelial neoplasia (PIN). C: Intense TRAP-1 staining in Gleason grade 3 cancer admixed with normal glands negative for TRAP-1 expression (arrow). D: Heterogeneous staining of TRAP-1 expression in Gleason grade 4 cancers (compare with C). E: Mixed staining of TRAP-1 expression in a case of intraductal carcinoma (IDC). Arrow, strongly stained IDC cell. Inset, TRAP-1 staining as punctate granules in the cytoplasm of an IDC cell. F, G: Heterogeneous TRAP-1 staining in Gleason grade 5 cancer with areas of weak $(\mathbf{F})$ or strong $(\mathbf{G})$ staining in separate cases. Magnifications, A, B $\times 400 ; \mathbf{C}, \mathbf{D}, \times 250 ; \mathbf{E}, \mathbf{F}, \mathbf{G}$ $\times 400$. Inset, $\times 400$.

population within lymph nodes (Figure 2, A and B), and bones (Figure 2, D-F). In contrast, non neoplastic constituents of lymph nodes and bones were unstained for TRAP-1 (Figure 2, D-F), and a nonbinding IgG did not stain metastatic cancer cells in either location (Figure 2C and not shown).

\section{Expression of TRAP-1 in Genetic Mouse Models} of Prostate Cancer

We next looked at the expression of TRAP-1 in genetic mouse models of prostate cancer. Immunohistochemical analysis of 22- to 26-week-old TRAMP mice revealed that

Table 1. Summary of TRAP-1 Staining Intensity in Normal Prostate or Prostate Cancer

\begin{tabular}{|c|c|c|c|c|c|}
\hline \multicolumn{6}{|c|}{ Staining intensity } \\
\hline Diagnosis & $\begin{array}{l}\text { Number } \\
\text { of cases }\end{array}$ & Negative $(-)$ & Negligible $(+)$ & Weak $(++)$ & $\begin{array}{c}\text { Strong } \\
(+++/++++)\end{array}$ \\
\hline Normal glands* & 17 & 5 & 9 & 3 & \\
\hline Normal glands ${ }^{\dagger}$ & 4 & 4 & & & \\
\hline High-grade PIN & 8 & & & 6 & 2 \\
\hline Gleason grade 3 & 15 & & 1 & 9 & 5 \\
\hline Gleason grade 4 & 8 & & 5 & 3 & \\
\hline Gleason grade 5 & 9 & & 4 & 3 & 2 \\
\hline
\end{tabular}

*Normal glands admixed with cancer.

+Normal glands in CZ and PZ samples collected at autopsy. 

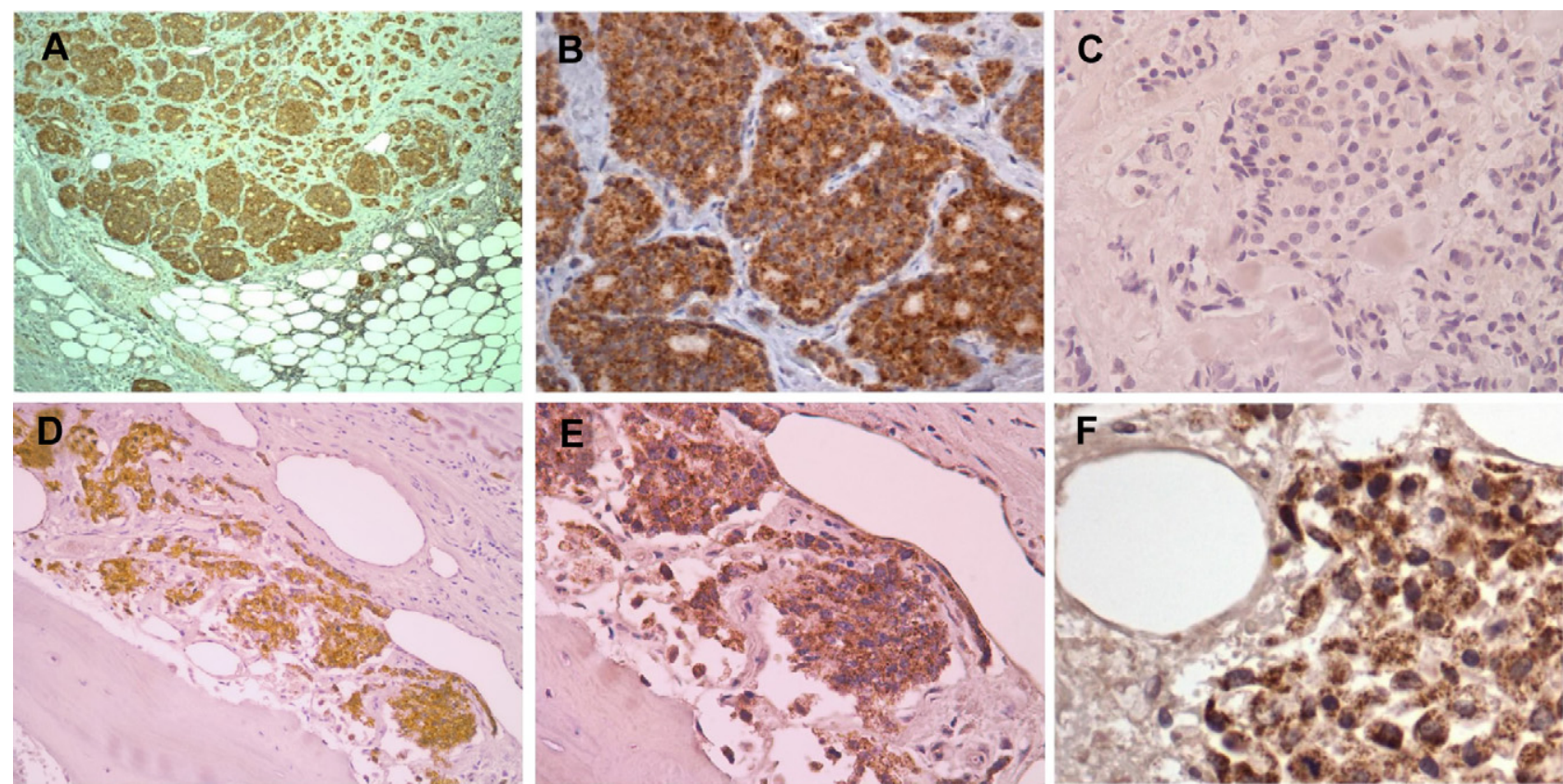

Figure 2. Expression of TRAP-1 in metastatic prostate cancer. Tissue sections from five independent cases of metastatic prostatic adenocarcinoma to lymph nodes $(\mathbf{A}, \mathbf{B})$ or bone $(\mathbf{C}-\mathbf{F})$ were stained with an antibody to TRAP-1 (A, B, D-F) or nonbinding IgG (C). All samples of metastatic prostate cancer were uniformly and strongly stained for TRAP-1 expression. Magnifications, $\times 100(\mathbf{A}, \mathbf{D}) ; \times 200(\mathbf{B}, \mathbf{C}, \mathbf{E}) ; \times 400(\mathbf{F})$.

TRAP-1 was abundantly and uniformly expressed in highgrade PIN lesions and invasive cancers (Figure $3 \mathrm{~A})$. The prostate samples from Pten ${ }^{p-1-}$ mice reviewed in this study did not contain foci of invasive cancer. However, high-grade PIN lesions in these specimens revealed heterogeneous staining for TRAP-1, ranging from weak to intensely positive (Figure $3 \mathrm{C}$ ). Similar to human samples, TRAP-1 staining of mouse lesions was seen as punctate cytoplasmic foci in all tumor cells (Figure 3, A and C). As control, nonbinding IgG did not stain prostatic transformed cells in TRAMP (Figure 3B) or Pten ${ }^{\text {Pc-l- }}$ (Figure 3D) lesions, by immunohistochemistry.

\section{TRAP-1-Mediated Cytoprotection in Prostate} Cancer

Consistent with its presence in primary and metastatic prostate cancer, in vivo, TRAP-1 was abundantly expressed in androgen-independent PC3 and DU145 prostate cancer cell lines, and to a lesser extent in androgendependent LNCaP cells, and their invasive counterpart, C4-2 cells, ${ }^{20}$ by Western blotting (Figure 4A). In contrast, nontransformed prostatic epithelial $\mathrm{BPH}-1$ cells had low levels of endogenous TRAP-1 (Figure 4A). To test a role of TRAP-1 in cytoprotection of prostatic epithelium, ${ }^{14}$ we
TRAP-1
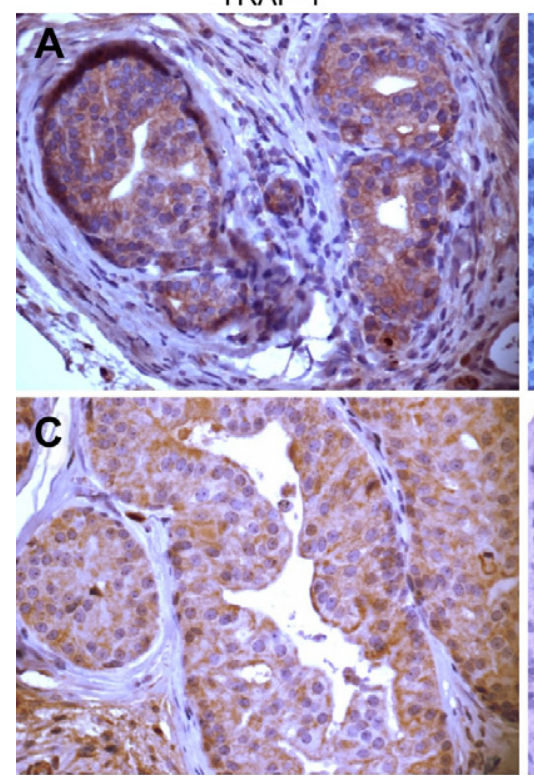

$\lg G$

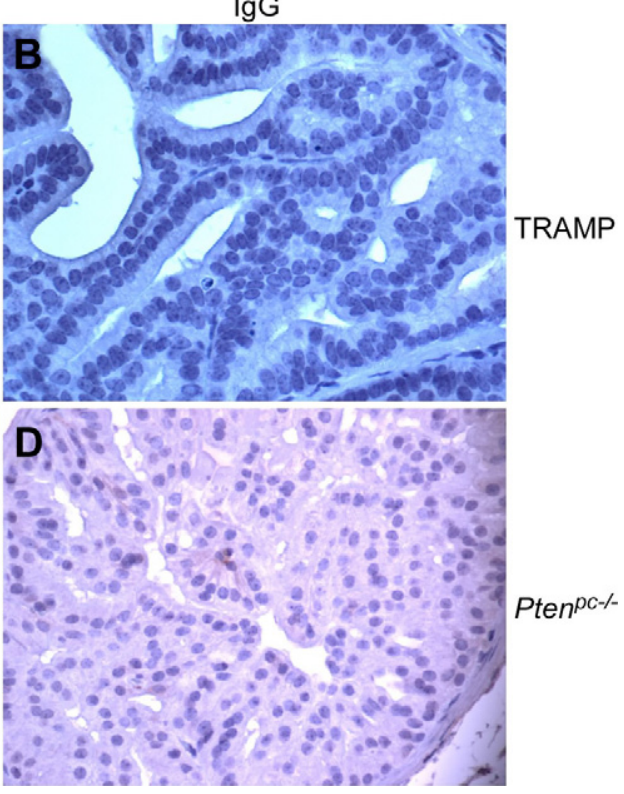

Figure 3. Expression of TRAP-1 in genetic mouse models of prostate cancer. Prostate tissue sections collected from TRAMP $(\mathbf{A}, \mathbf{B})$ or Pten $^{p c^{c-1}-}(\mathbf{C}, \mathbf{D})$ mice were stained with an antibody to TRAP-1 (A, C) or nonbinding IgG (B, D). Intense staining is evident in invasive cancer from a TRAMP mouse prostate (A). Heterogeneous TRAP-1 expression in a high-grade PIN lesion from the prostate of a Pten $n^{p c-1-}$ mouse (C), with strongly stained cells juxtaposed to negative or weakly stained cells. Four independent cases per model were examined with identical results. Magnification, $\times 200$. 
A

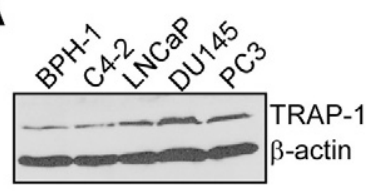

B

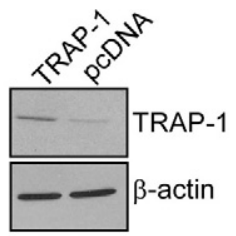

C

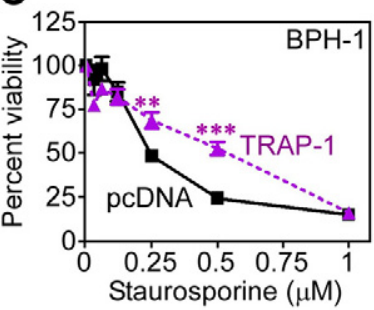

E

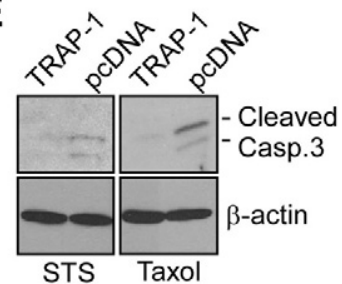

D

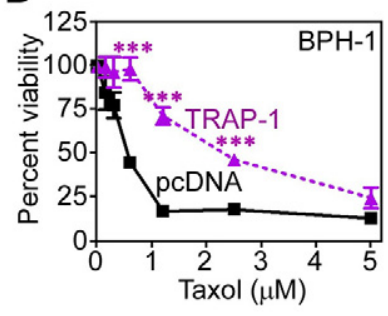

Figure 4. TRAP-1 cytoprotection in non-transformed prostatic epithelium A: Androgen-dependent (LNCaP) or -independent (C4-2B, PC3, DU145) prostate cancer cells or non-transformed prostatic epithelial (BPH-1) cells were analyzed by Western blotting. B: BPH-1 cells were transfected with control pcDNA or TRAP-1 cDNA, and analyzed by Western blotting. C-D: Transfected BPH-1 cells were treated with the indicated increasing concentrations of staurosporine (STS) (C), or taxol (D), and analyzed for changes in cell viability by MTT. ${ }^{* * *} P=0.0022$; ${ }_{* * * * *} P<0.0001$. Mean \pm SEM were calculated from $n=3$. The experiment was repeated twice with comparable results. E: BPH-1 cells transfected with pcDNA or TRAP-1 CDNA were exposed to the indicated cell death stimuli, and analyzed for differential expression of cleaved, ie, active caspase 3 fragments of 17 and 19 $\mathrm{kDa}$, by Western blotting.

next transfected $\mathrm{BPH}-1$ cells with control plasmid or TRAP-1 cDNA (Figure 4B), and examined their sensitivity to apoptosis. Treatment of control $\mathrm{BPH}-1$ transfectants with the broad cell death stimulus, STS, which is often used to induce mitochondrial cell death (Figure 4C), or the chemotherapeutic agent, taxol (Figure 4D), resulted in concentration-dependent loss of cell viability by MTT. In contrast, $\mathrm{BPH}-1$ cells transfected with TRAP-1 were significantly more resistant to STS- or taxol-induced cell death (Figure 4, C and D). Consistent with genuine modulation of apoptosis under these conditions, TRAP-1-expressing $\mathrm{BPH}-1$ cells exhibited reduced levels of cleaved, ie, active caspase 3 fragments of 17 and $19 \mathrm{kDa}$ after STS or taxol treatment, compared with pcDNA transfectants, by Western blotting (Figure 4E).

As a complementary approach, we next silenced TRAP-1 expression in prostate adenocarcinoma PC3 or DU145 cells by siRNA. Transfection of these cells with TRAP-1-directed siRNA significantly suppressed the expression of endogenous TRAP-1, whereas a control nontargeting siRNA had no effect on TRAP-1 levels, by Western blotting (Figure 5A). Under these conditions, siRNA knockdown of TRAP-1 in PC3 (Figure 5B), or DU145 (Figure 5C) cells resulted in increased sensitivity to STSinduced apoptosis, as compared with control siRNA transfectants, by MTT.

\section{TRAP-1 Expression Confers Sensitivity to Mitochondria-Targeted Hsp90 Antagonists}

To disable TRAP-1 cytoprotection in prostate cancer, we next used Gamitrinibs, a new class of combinatorial, mitochondria-targeted small molecule Hsp90 antagonists. ${ }^{16}$ A 6-hour exposure of androgen-independent prostate cancer DU145 cells to Gamitrinib-G3 or -G4 resulted in concentration-dependent loss of cell viability, whereas at this time point Gamitrinib-G2 had partial activity, and Gaminitrib-G1 or -TPP had negligible effect on cell viability, by MTT (Figure 6A, left panel). A more prolonged, 24 hours exposure of prostate cancer cells to all Gamitrinib variants resulted in further increased anticancer activity (Figure 6A, right panel). In contrast, unconjugated 17-AAG did not decrease prostate cancer cell viability within the same time intervals (Figure 6A). Similar results were obtained with androgen-dependent prostate cancer LNCaP cells, which exhibited comparable sensitivity to all Gamitrinib variants, but not unconjugated 17-AAG, after a 6-hour treatment, and complete loss of cell viability after a 24 hours exposure to the drug (Figure 6B).

At variance with these results, and consistent with their low level of endogenous TRAP-1, BPH-1 cells transfected with pcDNA were largely insensitive to Gamitrinib-induced killing, and only elevated concentrations of Gamitrinib-G4 >15 $\mu \mathrm{mol} / \mathrm{L}$ resulted in partial, 50\% reduction in cell viability (Figure 6C). Conversely, reintroduction of TRAP-1 in these cells conferred sensitivity to GamitrinibG4-induced apoptosis, quantitatively comparable with that of androgen-dependent (LNCaP) or -independent (DU145) prostate cancer cell types, by MTT (Figure 6, $A-C)$. In these experiments, unconjugated 17-AAG had no effect on $\mathrm{BPH}-1$ cell viability, in the presence or absence of TRAP-1 (Figure 6C).
A



B

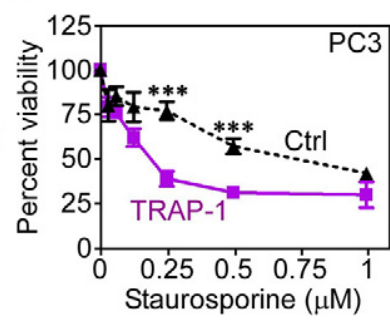

C



Figure 5. TRAP-1 promotes prostate cancer cell survival. A: PC3 or DU145 cells were transfected with non-targeting (Control, Ctrl) or TRAP-1-directed siRNA and analyzed after 48 hours by Western blotting. B-C: siRNA-transfected prostate cancer PC3 (B), or DU145 (C) cells were treated with increasing concentrations of staurosporine, and analyzed for changes in cell viability by MTT. Mean \pm SEM were calculated from $n=3$. The experiment was repeated twice with comparable results. ${ }^{* *} P=0.0015-0.0036 ;{ }^{* * *} P=$ $0.0007-<0.0001$. 


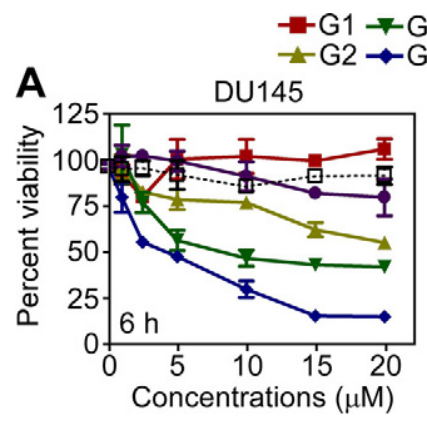

\section{$\rightarrow-T P P$}
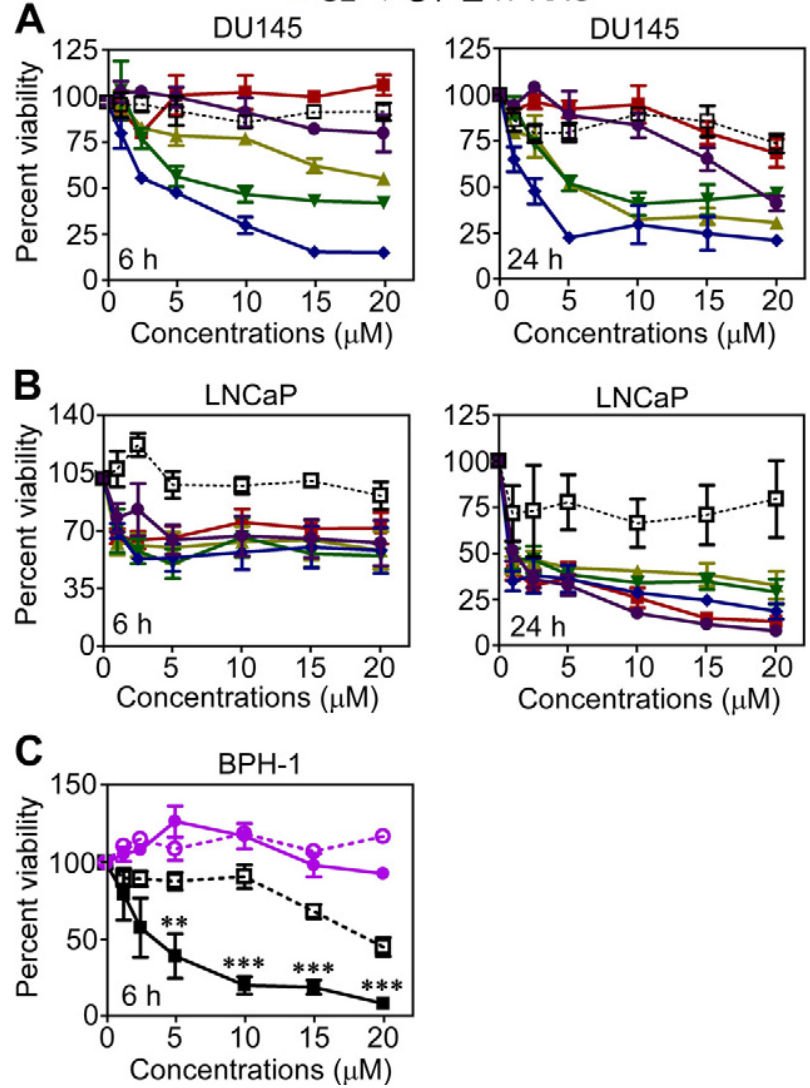

Figure 6. TRAP-1 confers sensitivity to Gamitrinib-induced cell killing. A, B: Androgen-independent DU145 (A), or androgen-dependent LNCaP (B) prostate cancer cells were treated with the indicated increasing concentrations of Gamitrinibs or unconjugated 17-AAG, and analyzed for cell viability by MTT after 6 hours (left panels) or 24 hours (right panels). C: BPH-1 cells were transfected with control (open symbols) or TRAP-1 (closed symbols) cDNA, treated with the indicated increasing concentrations of Gamitrinib-G4 (black) or unconjugated 17-AAG (purple), and analyzed by MTT after 6 hours. Mean \pm SEM were calculated from $n=3$. The experiment was repeated twice with comparable results. ${ }^{* * *} P=0.0084 ;{ }^{* * * * *} P<0.0001$

\section{Discussion}

In this study, we have shown that a mitochondrial Hsp90 homolog, TRAP- $1^{14}$ is abundantly and differentially expressed in localized and metastatic prostate cancer in humans, as well as genetic disease models in rodents. In contrast, in normal prostate or BPH glands, TRAP-1 was largely undetectable or minimally expressed. The sharp differential distribution of TRAP-1 in prostate cancer versus normal glands is in agreement with an earlier preliminary survey of different tumor types, where TRAP-1 was also differentially expressed in tumors of breast, lung, colon and pancreas, as compared with normal matched tissues. Functionally, TRAP-1 expression in prostate cancer conferred resistance to apoptosis, whereas targeting TRAP-1 with a new class of mitochondria-directed small molecule Hsp90 inhibitors, ie, Gamitrinibs, ${ }^{16}$ resulted in tumor cell death without affecting nontransformed prostatic epithelium.

Most epithelial malignancies, including prostate cancer, exhibit a higher anti-apoptotic threshold, which contributes to disease progression. ${ }^{21}$ However, how this pro- cess is regulated, especially with respect to mitochondrial integrity, has remained largely unknown. ${ }^{9}$ The data presented here support a recent model that molecular chaperones of the Hsp90 family, including TRAP-1, function as novel regulators of mitochondrial integrity in transformed cells, potentially by opposing the pore-forming function of cyclophilin $D^{14}$ in permeability transition. ${ }^{9}$ Consistent with this view, ${ }^{14}$ TRAP-1 has been shown to inhibit mitochondrial apoptosis induced by oxidative stress, ${ }^{22,23}$ in a pathway involving an activating phosphorylation by the mitochondrial kinase, PINK1. ${ }^{24}$ The ability of mitochondrial Hsp90 chaperones to oppose cyclophilin D activity, especially in response to oxidative stress, ${ }^{10,11}$ is probably critical to preserve neuronal survival, ${ }^{25}$ and it is possible that disparate malignancies, including prostate cancer (this study), commandeer this cytoprotective pathway to globally elevate a mitochondrial anti-apoptotic threshold. The resulting survival advantage for cancer cells would be uniquely suited to oppose oxidative stress-induced cell damage, which invariably accompanies tumor growth, in vivo.

The molecular basis for the sharp differential localization of Hsp90 and TRAP-1 to tumor versus normal mitochondria remains to be elucidated. One possibility is that tumor cells exhibit more avid mitochondrial import machinery than normal tissues, resulting in enhanced recruitment to mitochondria of nuclear-encoded gene products, including Hsp90 chaperones. Accordingly, TRAP-1 contains a genuine mitochondrial import sequence implicated in mitochondrial localization, ${ }^{26}$ and deregulated Akt signaling as often observed in prostate cancer, has been associated with increased expression and function of the Tom70 mitochondrial import receptor in tumor cells. $^{27}$ Here, elevated expression of TRAP-1 was observed in early prostatic lesions of Pten ${ }^{p c-/-}$ mice, suggesting that unbridled Akt activity under these conditions may contribute to the increased localization of TRAP-1 to mitochondria, in vivo, temporally preceding full blown transformation.

In the current study, histologically normal glands in the central zone and peripheral zone regions collected at autopsy were completely devoid of TRAP-1 expression, whereas some of the apparently histologically normal glands admixed within cancer exhibited negligible or weak staining for TRAP-1. The basis for these observations remains to be elucidated, but may reflect the acquisition of TRAP-1 cytoprotection in cytologically normal cells undergoing early neoplastic transformation. In this context, elevated levels of TRAP-1 were observed at the earliest stages of prostate tumorigenesis, and this expression persisted throughout the entire spectrum of Gleason grades of prostate cancer. A similar pattern was observed in prostate cancer metastases to lymph nodes and bones, altogether supporting the idea that TRAP-1 cytoprotection is exploited at all stages of prostate cancer. In clinical samples, TRAP-1 staining was uniformly present in grade 3 cancers, more heterogeneous in highergrade tumors, and again uniformly strong in metastases to lymph nodes and bone. This suggests that higher expression of TRAP-1 in subpopulations of high-grade localized cancers may confer an inherent advantage to 
further establish distant metastatic sites, as well as resistance to mainstay therapy, ie, taxol (this study). ${ }^{28}$ Interestingly, atrophic benign glands often exhibited increased TRAP-1 expression, which tended to localize to basal cells. These glands were not associated with inflammatory infiltrates characteristic of proliferative inflammatory atrophy, a proposed preneoplastic lesion. ${ }^{29}$ It is currently believed that the basal compartment of prostatic acini contains cells with progenitor/stem cell-like properties, ${ }^{30}$ and expression of TRAP-1 in this cellular compartment may reflect an enhanced cell survival mechanism potentially contributing to tissue regeneration.

Despite a better understanding of the molecular pathogenesis of prostate cancer, ${ }^{4}$ the therapeutic options for patients with advanced and metastatic disease remain limited, providing only small gains in overall survival. ${ }^{4}$ Here, disabling TRAP- 1 cytoprotection with a novel class of mitochondria-directed Hsp90 inhibitors, ie, Gamitrinibs $^{16}$ resulted in rapid and complete killing of androgen-dependent or -independent prostate cancer cell types, without affecting nontransformed prostatic epithelium. Mechanistically, Gamitrinibs activate cyclophilin Ddependent mitochondrial permeability transition in the tumor cells, resulting in loss of mitochondrial inner membrane potential, rupture of the outer membrane, and discharge of apoptogenic proteins, ie, cytochrome c, in the cytosol. ${ }^{16}$ Such direct mitochondriotoxic activity of Gamitrinibs is different from conventional chemotherapy or unconjugated Hsp90 inhibitors, which indirectly induce mitochondrial dysfunction, ${ }^{15}$ and are thus susceptible to compensatory survival mechanisms. The elevated degree of selectivity of Gamitrinibs for prostate cancer cells, compared with non-transformed prostatic epithelium, ie, $\mathrm{BPH}-1$ cells, bodes well for the further clinical development of these agents for use in humans. In addition to the differential quantitative expression in tumor versus normal mitochondria, ${ }^{16}$ it is also possible that the ATPase activity of TRAP-1 is qualitatively increased in transformed cells compared with normal tissues, thus similar to the paradigm of Hsp90, ${ }^{31}$ and further contributing to the potential tumor selectivity of Gamitrinibs.

In summary, we identified TRAP-1 as a novel marker of localized and metastatic prostate cancer, but not normal glands, required for prostate cancer cell viability, in vivo. Taken together with the preliminary safety of Gamitrinibs in preclinical studies, ${ }^{16}$ these data suggest that targeting mitochondrial TRAP-1 may provide a novel therapeutic approach for patients with advanced and metastatic prostate cancer. ${ }^{4}$

\section{Acknowledgment}

We thank Dr. Hong Wu for Pten ${ }^{p c-l-}$ mice.

\section{References}

1. Jemal A, Siegel R, Ward E, Hao Y, Xu J, Murray T, Thun MJ: Cancer statistics, 2008. CA Cancer J Clin 2008, 58:71-96

2. Carter HB, Ferrucci L, Kettermann A, Landis P, Wright EJ, Epstein JI, Trock BJ, Metter EJ: Detection of life-threatening prostate cancer with prostate-specific antigen velocity during a window of curability. J Natl Cancer Inst 2006, 98:1521-1527

3. Petrylak DP, Tangen CM, Hussain MH, Lara PN Jr, Jones JA, Taplin ME, Burch PA, Berry D, Moinpour C, Kohli M, Benson MC, Small EJ, Raghavan D, Crawford ED: Docetaxel and estramustine compared with mitoxantrone and prednisone for advanced refractory prostate cancer. N Engl J Med 2004, 351:1513-1520

4. Taichman RS, Loberg RD, Mehra R, Pienta KJ: The evolving biology and treatment of prostate cancer. J Clin Invest 2007, 117:2351-2361

5. Debes JD, Tindall DJ: Mechanisms of androgen-refractory prostate cancer. N Engl J Med 2004, 351:1488-1490

6. Mundy GR: Metastasis to bone: causes, consequences and therapeutic opportunities. Nat Rev Cancer 2002, 2:584-593

7. Majumder PK, Febbo PG, Bikoff R, Berger R, Xue Q, McMahon LM, Manola J, Brugarolas J, McDonnell TJ, Golub TR, Loda M, Lane HA, Sellers WR: mTOR inhibition reverses Akt-dependent prostate intraepithelial neoplasia through regulation of apoptotic and HIF-1-dependent pathways. Nat Med 2004, 10:594-601

8. Wei MC, Zong WX, Cheng EH, Lindsten T, Panoutsakopoulou V, Ross AJ, Roth KA, MacGregor GR, Thompson CB, Korsmeyer SJ: Proapoptotic BAX and BAK: a requisite gateway to mitochondrial dysfunction and death. Science 2001, 292:727-730

9. Green DR, Kroemer G: The pathophysiology of mitochondrial cell death. Science 2004, 305:626-629

10. Baines CP, Kaiser RA, Purcell NH, Blair NS, Osinska H, Hambleton MA, Brunskill EW, Sayen MR, Gottlieb RA, Dorn GW, Robbins J, Molkentin JD: Loss of cyclophilin $\mathrm{D}$ reveals a critical role for mitochondrial permeability transition in cell death. Nature 2005, 434:658-662

11. Nakagawa T, Shimizu S, Watanabe T, Yamaguchi O, Otsu K, Yamagata $\mathrm{H}$, Inohara H, Kubo T, Tsujimoto Y: Cyclophilin D-dependent mitochondrial permeability transition regulates some necrotic but not apoptotic cell death. Nature 2005, 434:652-658

12. Whitesell L, Lindquist SL: HSP90 and the chaperoning of cancer. Nat Rev Cancer 2005, 5:761-772

13. He L, Lemasters JJ: Regulated and unregulated mitochondrial permeability transition pores: a new paradigm of pore structure and function? FEBS Lett 2002, 512:1-7

14. Kang BH, Plescia J, Dohi T, Rosa J, Doxsey SJ, Altieri DC: Regulation of tumor cell mitochondrial homeostasis by an organelle-specific Hsp90 chaperone network. Cell 2007, 131:257-270

15. Isaacs JS, Xu W, Neckers L: Heat shock protein 90 as a molecular target for cancer therapeutics. Cancer Cell 2003, 3:213-217

16. Kang BH, Plescia J, Song HY, Meli M, Colombo G, Beebe K, Scroggins B, Neckers L, Altieri DC: Combinatorial drug design targeting multiple cancer signaling networks controlled by mitochondrial Hsp90. J Clin Invest 2009, 119:454-464

17. Cohen RJ, Wheeler TM, Bonkhoff H, Rubin MA: A proposal on the identification, histologic reporting, and implications of intraductal prostatic carcinoma. Arch Pathol Lab Med 2007, 131:1103-1109

18. Kaplan-Lefko PJ, Chen TM, Ittmann MM, Barrios RJ, Ayala GE, Huss WJ, Maddison LA, Foster BA, Greenberg NM: Pathobiology of autochthonous prostate cancer in a pre-clinical transgenic mouse model. Prostate 2003, 55:219-237

19. Wang S, Gao J, Lei Q, Rozengurt N, Pritchard C, Jiao J, Thomas GV, Li G, Roy-Burman P, Nelson PS, Liu X, Wu H: Prostate-specific deletion of the murine Pten tumor suppressor gene leads to metastatic prostate cancer. Cancer Cell 2003, 4:209-221

20. Thalmann GN, Anezinis PE, Chang SM, Zhau HE, Kim EE, Hopwood VL, Pathak S, von Eschenbach AC, Chung LW: Androgen-independent cancer progression and bone metastasis in the LNCaP model of human prostate cancer. Cancer Res 1994, 54:2577-2581

21. Huang $X$, Zhang $X$, Farahvash B, Olumi AF: Novel targeted proapoptotic agents for the treatment of prostate cancer. J Urol 2007, 178:1846-1854

22. Hua G, Zhang Q, Fan Z: Heat shock protein 75 (TRAP1) antagonizes reactive oxygen species generation and protects cells from granzyme M-mediated apoptosis. J Biol Chem 2007, 282:20553-20560

23. Montesano Gesualdi N, Chirico G, Pirozzi G, Costantino E, Landriscina M, Esposito F: Tumor necrosis factor-associated protein 1 (TRAP-1) protects cells from oxidative stress and apoptosis. Stress 2007, 10:342-350

24. Pridgeon JW, Olzmann JA, Chin LS, Li L: PINK1 Protects against oxidative stress by phosphorylating mitochondrial chaperone TRAP1. PLoS Biol 2007, 5:e172 
25. Gandhi S, Wood-Kaczmar A, Yao Z, Plun-Favreau H, Deas E, Klupsch K, Downward J, Latchman DS, Tabrizi SJ, Wood NW, Duchen MR, Abramov AY: PINK1-associated Parkinson's disease is caused by neuronal vulnerability to calcium-induced cell death. Mol Cell 2009, 33:627-638

26. Felts SJ, Owen BA, Nguyen P, Trepel J, Donner DB, Toft DO: The hsp90-related protein TRAP1 is a mitochondrial protein with distinct functional properties. J Biol Chem 2000, 275:3305-3312

27. Wright GL, Maroulakou IG, Eldridge J, Liby TL, Sridharan V, Tsichlis PN, Muise-Helmericks RC: VEGF stimulation of mitochondrial biogenesis: requirement of AKT3 kinase. FASEB J 2008, 22:3264-3275

28. Silvestris N, Leone B, Numico G, Lorusso V, De Lena M: Present status and perspectives in the treatment of hormone-refractory prostate cancer. Oncology 2005, 69:273-282

29. De Marzo AM, Platz EA, Sutcliffe S, Xu J, Gronberg H, Drake CG Nakai $Y$, Isaacs WB, Nelson WG: Inflammation in prostate carcinogenesis. Nat Rev Cancer 2007, 7:256-269

30. Goldstein AS, Lawson DA, Cheng D, Sun W, Garraway IP, Witte ON: Trop2 identifies a subpopulation of murine and human prostate basal cells with stem cell characteristics. Proc Natl Acad Sci USA 2008, 105:20882-20887

31. Kamal A, Thao L, Sensintaffar J, Zhang L, Boehm MF, Fritz LC, Burrows FJ: A high-affinity conformation of Hsp90 confers tumour selectivity on Hsp90 inhibitors. Nature 2003, 425:407-410 